\title{
Risk of Radiation Induced Carotid Artery Stenosis in Supraclavicular Lymph Node Irradiation in Breast Cancer Patients
}

\author{
Reham Abdulmoniem 1,2, Yasser Bayoumi1 ${ }^{1}$ Mushabbab Al Asiri², Reem Zatar ${ }^{3}$, \\ Abdullah Al Amro², Majed Mosely3, Mohamed Hamada3 ${ }^{3}$ Eyad Al Saeed ${ }^{4}$, \\ Abdulrahman Al Hadab'2, Mutahir A. Tunio² \\ ${ }^{1}$ Radiation Oncology Department, National Cancer Institute, Cairo University, Giza, Egypt \\ ${ }^{2}$ Radiation Oncology Department, Comprehensive Cancer Center, King Fahad Medical City, Riyadh, KSA \\ ${ }^{3}$ Medical Physics Department, Comprehensive Cancer Center, King Fahad Medical City, Riyadh, KSA \\ ${ }^{4}$ King Saud University, Riyadh, KSA \\ Email: dr.reham71@hotmail.com
}

Received 6 November 2013; revised 6 December 2013; accepted 13 December 2013

Copyright (C) 2014 by authors and Scientific Research Publishing Inc.

This work is licensed under the Creative Commons Attribution International License (CC BY).

http://creativecommons.org/licenses/by/4.0/

(c) (i) Open Access

\begin{abstract}
Background: The risk of carotid vessel stenosis is high after radiotherapy (RT) to neck; however, carotid vessels are unavoidable during supraclavicular (SC-RT) in adjuvant treatment of breast cancer (BC). However, carotid vessels RT dose is less well-understood. We aimed to determine the carotid vessels doses received by different techniques for SC-RT. Materials and Methods: Thirty consecutive $\mathrm{BC}$ patients were planned to receive $\mathrm{SC}-\mathrm{RT}$ and breast after breast conserving surgery (BCS) after taking $5 \mathrm{~mm}$ image slices on computed tomography (CT) simulation. Organs at risk (OAR) including carotid vessels, lungs, spinal cord alongwith clinical target volume (CTV) for primary tumor and lymph nodes were delineated. Comparative analysis was done between two treatment plans; three-field conformal radiation therapy (3F-CRT) and four-field conformal RT (4F-CRT) for CTV and carotid vessels doses. Results: The 4F-CRT was found better than 3F-3DCRT regarding coverage and homogeneity of the CTV volume. The $98 \%$ of the CTV was covered by $84 \%$ dose in 3F-CRT and 95\% in 4F-CRT (p 0.001). The carotid vessels maximum dose in the in 3F-CRT was $54.5 \pm 1.3 \mathrm{~Gy}$ compared to $51.6 \pm 1.3 \mathrm{~Gy}$ in 4F-CRT (p 0.014), however the mean dose did not differ significantly ( $p$ value 0.8 ). The ipsilateral lung dose did not differ between the two techniques ( $p$ Value 0.9). Conclusion: The 4F-CRT plan was found better than 3F-CRT in CTV coverage with minimal dose to the carotid vessel and without significant higher dose to the ipsilateral lung.
\end{abstract}

\section{Keywords}

Breast Cancer; Supraclavicular Radiotherapy; Carotid Vessel Dose 


\section{Introduction}

Regional supraclavicular radiotherapy (SC-RT) is generally considered for the breast cancer (BC) patients with tumor size $\geq 5 \mathrm{~cm}$ and or $\geq 4$ involved axillary lymph nodes (LN). Recent data have shown locoregional benefit of SC-RT in patients with one to three involved axillary LN [1] [2]. However adjuvant radiotherapy trials failed to demonstrate any overall survival benefit for radiotherapy (RT) in BC because of attributed risk of vascular morbidity and mortality [3].

The radiobiological basis of radiation induced vasculopathy is that it mainly affects the small vessels rather than the larger ones. However, recently emerging data on RT in head and neck cancer patients have shown an increased frequency of stroke or transient ischemic attack (TIA) secondary to carotid vessels stenosis [4]-[7]. The carotid stenosis following RT is dose dependent as well as other associated co-morbid conditions like age, hypertension, smoking, diabetes mellitus and hypercholestremia [8]. In SC-RT in BC patients, the carotid vessels, by virtue of their proximity CTV, are included in the radiation fields while targeting CTV. With advent of novel techniques in past two decades like three dimensional conformal radiation therapy (3DCRT), it is possible to give maximum dose to tumor and minimal doses to adjacent organs at risks (OAR) like lung, spinal cord and heart. Similarly, carotid vessels can be delineated as one of OAR during SC-RT in BC patients based on evidence of RT induced carotid stenosis.

The purpose of our study is to evaluate the carotid dose in the most world widely used breast irradiation techniques; 3F-CRT and 4F-CRT.

\section{Materials and Methods}

After taking institutional ethical committee (IRB) approval and informed consent, thirty consecutive BC patients were included in the study for adjuvant RT after BCS in following manner.

\subsection{CT Simulation}

At the time of simulation, all patients were first positioned supine on breast board with head turned to opposite direction of treatment side and with both arms placed above the head. A single isocenter was chosen at the level of match line between the SC and breast below the medial end of clavicle. During simulation, a reference transverse plane was identified by laser light alignment at the level of the breast areola. CT images were obtained with a high-speed 16-slice helical scanner at $5 \mathrm{~mm}$ slices through the region of interest.

\subsection{Target Definition}

Breast, SC-LN, level III axillary LN ipsilateral carotid vessel, spinal cord, heart, and both lungs were contoured on each CT cut as shown in Figures $1(\mathrm{~A})$ and (B).

The level III axillary LN clinical target volume (CTV) was delineated superiorly by the insertion of the pectoralis minor muscle on the coracoids process, anteriorly by the posterior border of the pectoralis minor muscle, posteriorly by the ribs and intercostal muscles, and laterally by the medial border of the pectoralis minor muscle. We defined the medial and inferior limits by the edge of the visible axillary fat plane [9]. For SC-RT, CTV was defined medially by the lateral edge of the trachea excluding the thyroid gland, superiorly by the inferior edge of the cricoid cartilage, anteriorly by the posterior border of the sternocleidomastoid muscle, postero-laterally by the anterior border of the anterior scalene muscle, and postero-medially to include the carotid artery and jugular vein. At the inferior aspect of the SC-LN, the anterior border of the subclavian vessels forms the posterior border of CTV [9].

The CTV of SC-LN and level III axillary LNs were expanded uniformly by $0.5 \mathrm{~cm}$ to create a regional nodal planning target volume (LN-PTV).

\subsection{Radiation Techniques}

Two plans were generated for each patient by the same planner using Eclipse “Aria version” treatment planning software (Varian Medical Systems, Palo Alto, California). A dose of 50 Gy in 25 fractions, delivered was prescribed to the breast and regional lymph nodes. A dose volume histogram is created to check the LN-PTV coverage and the dose to OAR for each plan. 

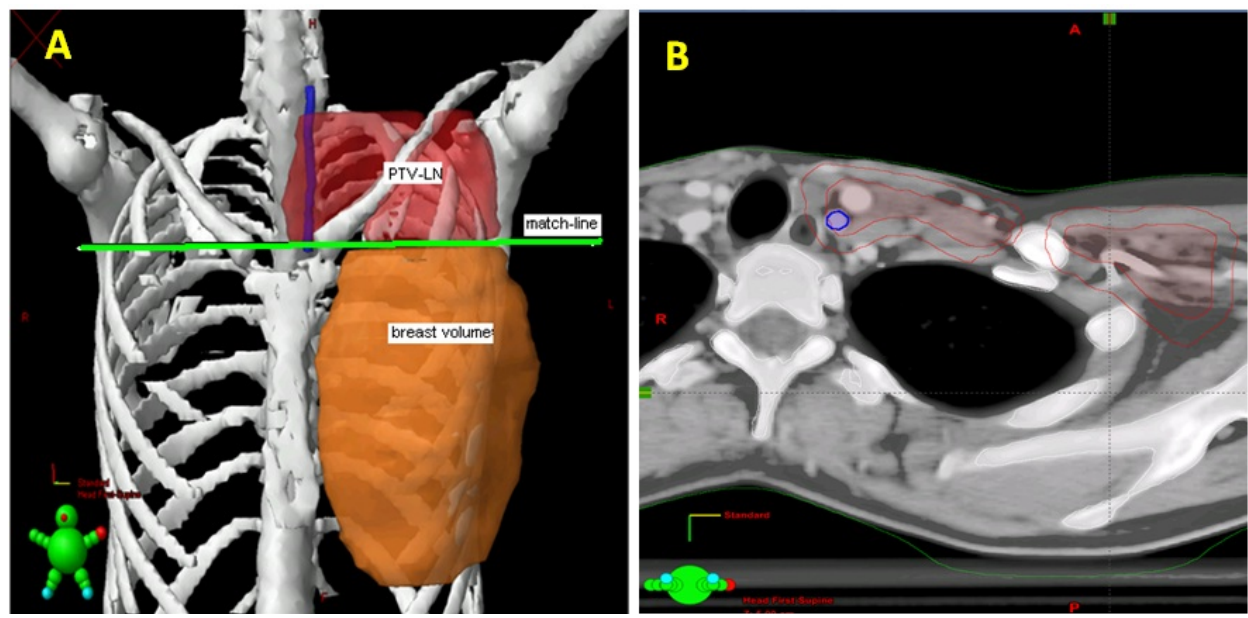

Figure 1. Three dimensional view and CT cut showing the target delineation (A) 3D view showing the PTV-LN, breast and match line between them and (B) CT cut showing the PTV-LN with CTV-LN inside in red color and carotid vessel in blue color.

\subsubsection{Three-Field Conformal RT Plan (3F-CRT)}

Opposed tangent fields were designed to encompass the contoured breast as follows: the superior edge of the field was defined by the inferior extent of the LN-PTV which correspond to the clinically chosen isocenter. The inferior edge was placed at $2 \mathrm{~cm}$ below the infra-mammary fold. The anterior border designed to be outside the body by $2 \mathrm{~cm}$. The angle of tangential fields is chosen to avoid as much as possible of the lung. Dynamic wedges were used to maintain homogeneity within $\pm 10 \%$.

A single AP was matched to the superior border of the tangential fields to encompass the LN-PTV. A small gantry angle of 5 - 15 degrees was applied to avoid the spinal cord. Dose was prescribed to the LN-PTV to allow $95 \%$ iso-dose curve covering the volume. Wedges were used to have homogeneity as per each case Figure 2.

\subsubsection{Four-Field Conformal RT Plan (4F-CRT)}

The same plan of 3F-CRT was used with adding posterior opposing field to AP field. Differential weighing between AP/PA fields was used with choosing 18MV energy for the PA field to attain homogeneity and conformity Figure 2.

\subsection{Statistical Analysis}

LN-PTV, ipsilateral lung and carotid vessel doses in the two treatment plans were evaluated. Statistical package for social science version 16.0 (SPSS; 16.0) was used for data analysis. Minimum, maximum, mean and standard deviations were used for quantitative data description. Statistical analysis was performed using one-way ANOVA to detect differences among the 2 plans. For those parameters with significant differences, a two-tailed paired student t-test was subsequently used to compare mean values among the different groups. p-value 0.05 was considered statistically significant.

\section{Results}

Comparative analysis according dose characteristics in both treatment plans is given in Table 1.

The 3F-CRT plans showed mean minimum dose to SC-CTV $3988 \pm 504$ cGy (range: 2709 - 4558) compared to mean minimum to SC-CTV $4463 \pm 401$ cGy (range: 3063 - 4926) for 4F-CRT. The SC-CTV maximum dose ranged from 5301 cGy to 5810 cGy in the 3F-CRT plan with mean maximum dose $5518 \pm 144$ cGy, while the 4F-CRT plan showed range of 5130 cGy to 5511 cGy and mean maximum dose $5333 \pm 111$ cGy. The difference between both techniques regarding the SC-CTV maximum dose was highly significant (p 0.001). The SC-CTV mean dose range in the 3F-CRT plan was 4750 - 5190 (mean $4942 \pm 109$ cGy) compared to 4890 - 5270 (mean $5051 \pm 89$ cGy). The difference in the mean dose was significant (p 0.02).

The iso dose curve covering the $98 \%$ of the volume ranges from $70 \%$ - $97 \%$ (mean $84 \%$ ) in the 3F-CRT plan 

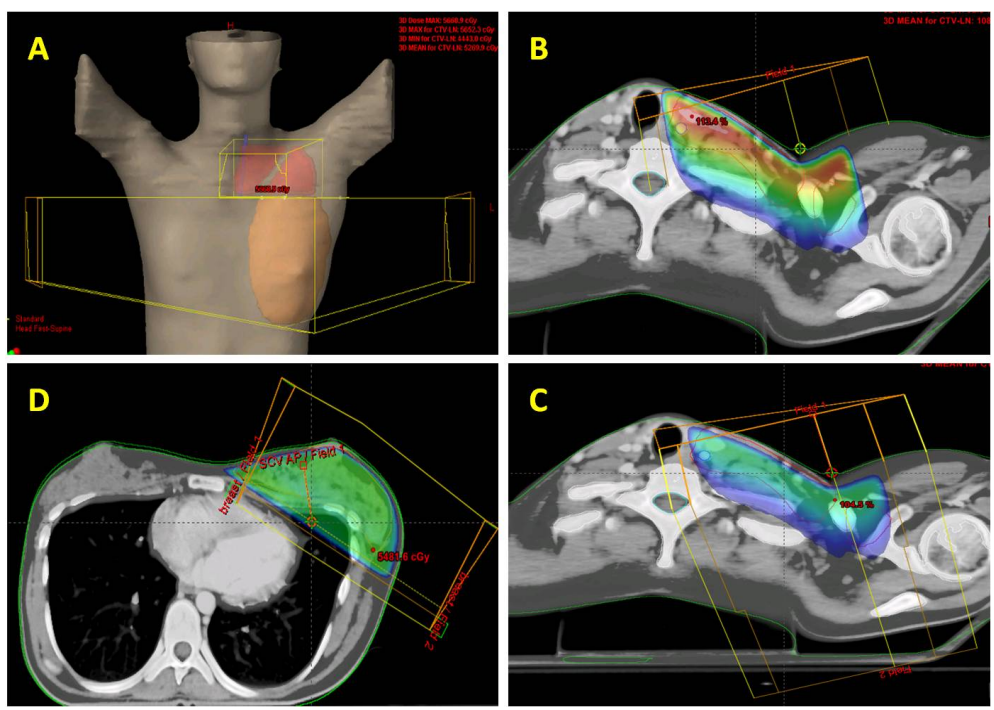

Figure 2. Treatment plans showing beams design and dose distributions for different techniques (A) The beam design, (B) Isodose distribution for 3FCRT at the level of SCV-LN, (C) Isodose distribution for 4F-CRT at the level of SCV-LN and (D) Isodose distribution at breast level for 2 tangential fields.

Table 1. The LN-PTV, ipsilateral lung and carotid vessel dose difference in 3F-CRT and 4F-CRT.

\begin{tabular}{|c|c|c|c|}
\hline \multirow{2}{*}{ Variable } & \multicolumn{2}{|c|}{ Technique } & \multirow{2}{*}{$\mathrm{p}$-value } \\
\hline & 3F-CRT & 4F-CRT & \\
\hline $\begin{array}{l}\text { SC-CTV max dose } \\
\bullet \quad \text { Dose range } \\
\bullet \quad \text { Mean } \pm \text { SD }\end{array}$ & $\begin{array}{c}5301-5810 \\
5518 \pm 144\end{array}$ & $\begin{array}{c}5130-5511 \\
5333 \pm 111\end{array}$ & 0.001 \\
\hline $\begin{array}{lr}\text { SC-CTV min dose } \\
\bullet \quad \text { Dose range } \\
\bullet \quad \text { Mean } \pm \text { SD }\end{array}$ & $\begin{array}{c}2709-4558 \\
3988 \pm 504\end{array}$ & $\begin{array}{c}3063-4926 \\
4463 \pm 401\end{array}$ & 0.01 \\
\hline $\begin{array}{l}\text { SC-CTV mean dose } \\
\bullet \quad \text { Dose range } \\
\bullet \quad \text { Mean } \pm \text { SD }\end{array}$ & $\begin{array}{c}4750-5190 \\
4942 \pm 109\end{array}$ & $\begin{array}{c}4890-5270 \\
5051 \pm 89\end{array}$ & 0.023 \\
\hline $\begin{array}{l}\text { SC-CTV } \text { D }_{98 \%}{ }^{*} \\
-\quad \text { Dose range } \\
\bullet \quad \text { Mean } \pm \text { SD }\end{array}$ & $\begin{array}{l}70 \%-97 \% \\
84 \% \pm 11 \%\end{array}$ & $\begin{array}{c}90 \%-99 \% \\
95 \% \pm 4 \%\end{array}$ & 0.001 \\
\hline $\begin{array}{cc}\text { Ipsilateral lung } \% \mathrm{~V}_{20 \mathrm{~Gy}}{ }^{* *} \\
\bullet \quad \text { Volume range } \\
\bullet \quad \text { Mean } \pm \mathrm{SD}\end{array}$ & $\begin{array}{c}12 \%-28 \% \\
20 \% \pm 3.8 \%\end{array}$ & $\begin{array}{c}12 \%-30 \% \\
21 \% \pm 4.3 \%\end{array}$ & 0.9 \\
\hline $\begin{array}{l}\text { Carotid max dose } \\
\bullet \quad \text { Dose range } \\
\bullet \quad \text { Mean } \pm \text { SD }\end{array}$ & $\begin{array}{c}5230-5661 \\
5454 \pm 131\end{array}$ & $\begin{array}{c}4874-5285 \\
5159 \pm 112\end{array}$ & 0.014 \\
\hline $\begin{array}{c}\text { Carotid mean dose } \\
\bullet \quad \text { Dose range } \\
\bullet \quad \text { Mean } \pm \text { SD }\end{array}$ & $\begin{array}{c}4356-5467 \\
5026 \pm 818\end{array}$ & $\begin{array}{c}4613-5121 \\
4966 \pm 128\end{array}$ & 0.3 \\
\hline
\end{tabular}

compared to 90\% - 99\% (mean 95\%) in the 4F-CRT. The difference was highly significant (p 0.001).

The ipsilateral lung dose was not affected significantly by adding the posterior field. The percent volume received 20 Gy ranged from $12 \%$ - 28\% (mean 20\% $\pm 3.8 \%$ ) in the 3F-CRT compared to $12 \%$ - 30\% (mean 21\% $\pm 4.3 \%$ ) in the 4 F-CRT technique (p 0.9). 
The maximum carotid dose ranged from 5230 cGy to 5661 cGy in the 3F-CRT plan compared to 4874 cGy to 5285 cGy in the 4F-CRT plan Figure 3.

The mean maximum dose of the carotid vessel was $5454 \pm 131$ for the 3F-CRT plan and $5159 \pm 112$ for the 4F-CRT plan with statistical significant difference (p value 0.014). The mean dose for the carotid vessel ranged from 2056 cGy to 5467 cGy (mean $5026 \pm 818$ ) for the 3F-CRT however, it ranged from 4613 cGy to 5121 (mean dose $4966 \pm 128$ ) in the 4F-CRT plan. There was no significant difference between the two studied plans regarding the mean carotid dose ( $\mathrm{p}$ value 0.8 ).

\section{Discussion}

Adjuvant radiation for breast cancer apart from improving loco regional control proved to have overall survival benefit, however, the serious late effects of radiation including the stroke probability could mask this benefit [3].

Many factors are well known to be risk factors for stroke and vascular stenosis including age, diabetes mellitus, hypertension, hypercholesterolemia and smoking [8]. Many studies have shown the radiation as one of causative factor for vasculopathy. Lam et al. reported 30\% of the nasopharyngeal cancer patients treated with radical radiotherapy developed significant common and internal carotid arteries stenosis compared to the pre radiotherapy [6].

The 15 year cumulative risk of carotid vessel stenosis in a 367 head and neck cancer patients reported to be $12 \%$ in Dorresteijn et al. study [7]. However, risk of stroke in BC patients treated with adjuvant SC-RT was found similar to non-irradiated BC patients with 10-year actuarial freedom from hospitalization for stroke was $91 \%$ for non-irradiated patients versus $89.5 \%$ for irradiated patients (p 0.9) [10].

Apart from this controversy, Chung et al. studied 16 BC patients by MRI before and after SC-RT to determine the frequency of narrowing of the carotid arterial lumen. All the patients received more than $40 \mathrm{~Gy}$ and all of them showed MRI vascular changes. When the MRI changes correlated with stenosis, it was found that $56 \%$ of patients had carotid artery stenosis. The differences between the pre and post radiation scans were statistically significant $(\mathrm{p}<0.05)$ for one reader and $(\mathrm{p}<0.0001)$ for another reader [11].
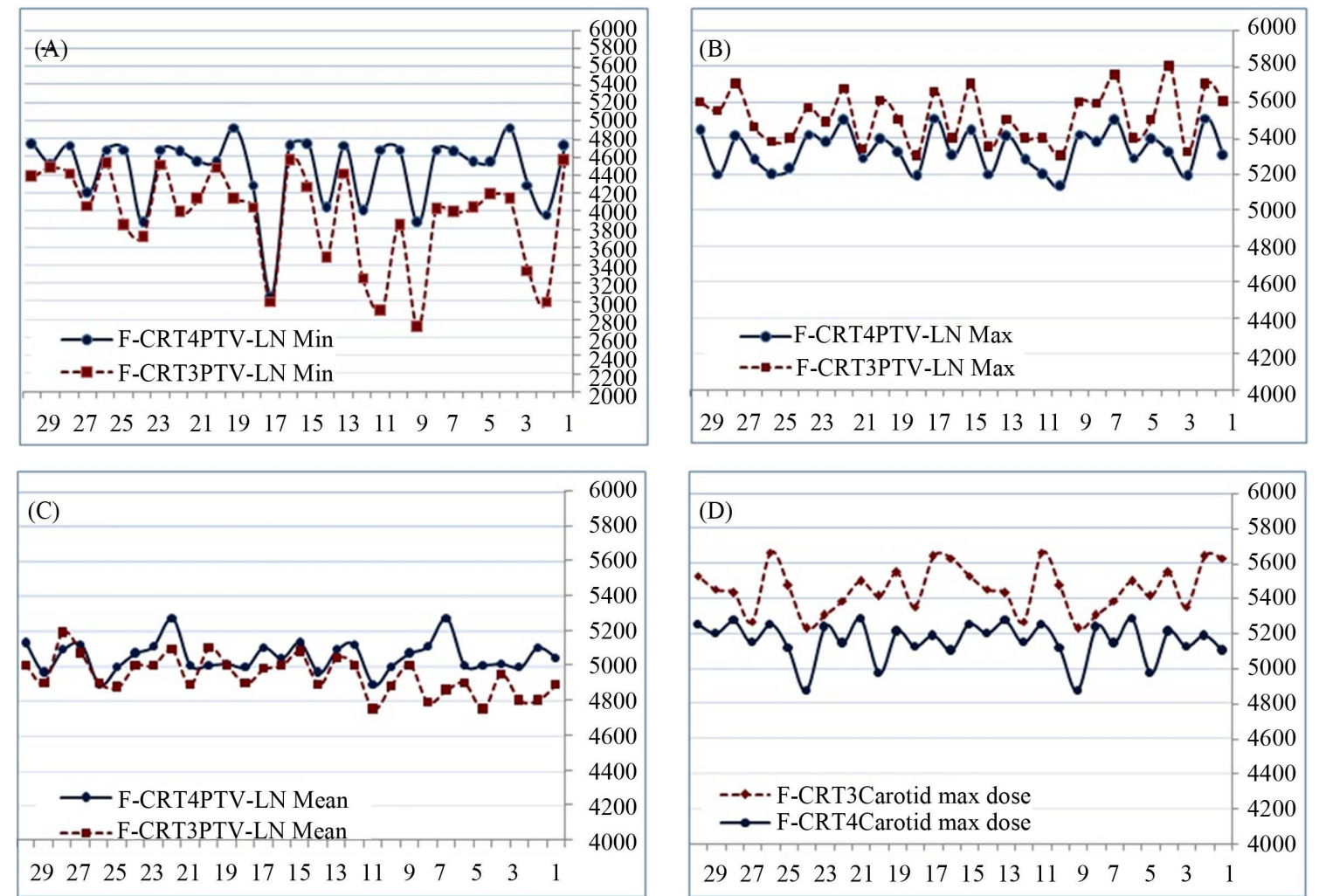

Figure 3. The dose difference between 3F-CRT and 4F-CRT for each patient. (A) PTV-LN minimum dose, (B) PTV-LN maximum dose, (C) PTV-LN mean dose and (D) carotid vessel maximum dose. 
Pathogenesis of radiation induced vascular stenosis start with development of degeneration of the media of large vessels followed by fibrosis, which appears at 2 months to 2 years after radiation. With time, the intima; just above media also starts a disruption. These effects cause irregularity of the surface of the large vessels which enhance the increase of intima media thickness (IMT) and atherosclerosis [12] [13].

The small vessels like the vasa vassorum are more susceptible for radiation induced damage as radiation inhibits capillary and vascular remodeling. Occlusion of the vasa vasorum may also be important in the late development of fibrotic and degenerative changes in irradiated vessels [14].

Radiation vasculopathy is thought to be both time and dose dependent, so one would expect that the larger the dose, the greater the effect and the longer the time after exposure, the more severe the expected stenosis [8].

The dose effect of the transient ischemic attacks and vascular stenosis is not well known. Elerding et al. showed that RT to head \& neck and lymphoma patients was associated with abnormal angiogram of the carotid in $25 \%$ of cases. They did not differentiate between lymphoma cases which receive maximum 40Gy and head \& neck cases receiving 60 - 70 Gy (13). These findings were consistent with Hull et al. results who reported the median SC-RT dose of 38 Gy (range, 30 - 57 Gy) for carotid stenosis [15]. Also, Graus et al. proposed that, the minimal dose for RT induced damage of the cervical arteries was assumed to be 41 Gy [16]. Bilora et al. showed increased IMT of the arterial wall in Hodgkin and non-Hodgkin patients received RT compared to matched controls [17] [18]. Gianocolo et al. confirmed these findings on 19 patients received neck irradiation and most of them had Hodgkin disease. The mean dose received was $41.2 \pm 15.6$ Gy (range: 25 - 70 Gy). Common carotid IMT was measured with echo-color Doppler. The data compared to 19 matched non-irradiated controls with the same risk factors. Although the difference did not reach statistical significance $(p=0.1)$, but the irradiated young patients (age $\leq 52$ years) had IMT measurements higher $(0.54 \pm 0.08 \mathrm{~mm})$ than the non-irradiated young patients $(0.49 \pm 0.14 \mathrm{~mm})$. The mean carotid IMT increased with increasing doses of radiation to the neck with p value 0.04 [19].

In view of the previous data, efforts shall be made to keep the dose to the carotid vessels minimum as much as possible especially in cases where prescribed dose exceeds $50 \mathrm{~Gy}$ or above. The different radiotherapy techniques have been used to treat the SC-RT in BC patients with variation in CTV coverage, excessive normal tissues irradiation, or producing undesirable hot spots. Current study was performed using CT based treatment planning. The 3F-CRT plans showed inadequate coverage of the SC-CTV and higher carotid doses as compared to 4F-CRT. Our findings are consistent with results of Jephcott et al. who evaluated 10 patients with BC. The axillary and SCV LN volumes in addition to the brachial plexus were contoured. Four plans for each patient were created including single AP, AP/PA, anterior field with PA boost and AP with PA boost. The AP plan gave poor PTV coverage in $60 \%$ of cases. The AP-PA provided good PTV coverage and minimal hot spots [20]. Wang et al. compared AP field, AP/PA and IMRT for SCV RT. Apart from the IMRT which was better regarding the volumes that received $105 \%$, $80 \%$, and $30 \%$ of prescribed dose outside the delineated volume, the AP/PA was found better than AP for CTV coverage and this study concluded that CT treatment planning with dose optimization around delineated target volumes should become standard for radiation treatments to avoid unnecessary hot spots [21].

In general, there is misconception that adding posterior field to the SC-RT is associated with increased lung dose. We did not see any difference in lung doses. The V20Gy of the ipsilateral lung was $21 \%$ in the $4 \mathrm{~F}-\mathrm{CRT}$ and 20\% in 3F-CRT (p 0.9). Lomax et al. reported the similar conclusions [22]. However, we believe that using V20Gy which was not reported in Lomax data is far better than using the mean lung doses from radiobiological point of view. By comparing 3 field plans of Vitolo et al. [23] with 3F-CRT in our series, we found that the mean dose to the carotid vessels was $98 \%$ compared to $100 \%$ respectively and the maximum dose $112 \%$ and $109 \%$ respectively, which confirms our results that the dose to the carotid vessels is relatively high by using single anterior field to SC-CTV.

\section{Conclusion and Recommendation}

The potential for stroke is well-recognized in patients with neck irradiation. Hypertension, diabetes mellitus, hypercholesterolemia and obesity are known to exacerbate the severity and speed of atherosclerosis.

We recommend optimizing the indications and technique for SC-CTV irradiation. The carotid vessels should be considered as OAR and should be delineated especially in high risk patients for stroke with associated comorbidities. The AP/PA for the SC-RT is better than the AP field regarding the dose to the carotid vessels and 
even better for target volume coverage. Using more sophisticated techniques is encouraged to have better conformal treatment.

Effective management of other modifiable risk factors with appropriate changes in lifestyle is highly recommended for patients with BC received radiation and hormonal treatment. In addition to that, routine follow-up for high risk patients for radiation induced carotid artery stenosis is advisable.

\section{References}

[1] Whelan, T.J., Olivotto, I., Ackerman, J., et al. (2011) NCIC-CTG MA.20: An Intergroup Trial of Regional Nodal Irradiation in Early Breast Cancer. Journal of Clinical Oncology, 29, LBA1003.

[2] Truong, P.T., Jones, S.O., Kader, H.A., et al. (2009) Patients with t1 to t2 Breast Cancer with One to Three Positive Nodes Have Higher Local and Regional Recurrence Risks Compared with Node-Negative Patients after Breast-Conserving Surgery and Whole-Breast Radiotherapy. International Journal of Radiation Oncology • Biology • Physics, $\mathbf{7 3}$, 357-364. http://dx.doi.org/10.1016/j.ijrobp.2008.04.034

[3] Early Breast Cancer Trialists' Collaborative Group (2000) Favourable and Unfavorable Effects on Long-Term Survival of Radiotherapy for Early Breast Cancer: An Overview of the Randomized Trials. Lancet, 355, 1757-1770. http://dx.doi.org/10.1016/S0140-6736(00)02263-7

[4] Dubec, J.J., Munk, P.L., Tsang, V., et al. (1998) Carotid Artery Stenosis in Patients Who Have Undergone Radiation Therapy for Head and Neck Malignancy. The British Journal of Radiology, 71, 872-875.

[5] Cheng, S.W., Wu, L.L., Ting, A.C., et al. (1999) Irradiation-Induced Extracranial Carotid Stenosis in Patients with Head and Neck Malignancies. The American Journal of Surgery, 178, 323-328. http://dx.doi.org/10.1016/S0002-9610(99)00184-1

[6] Lam, W.W., Leung, S.F., So, N.M., et al. (2001) Incidence of Carotid Stenosis in Nasopharyngeal Carcinoma Patients after Radiotherapy. Cancer, 92, 2357-2363. http://dx.doi.org/10.1002/1097-0142(20011101)92:9<2357::AID-CNCR1583>3.0.CO;2-K

[7] Dorresteijn, L.D., Kappelle, A.C., et al. (2002) Increased Risk of Ischemic Stroke after Radiotherapy on the Neck in Patients Younger Than 60 Years. Journal of Clinical Oncology, 20, 282-288. http://dx.doi.org/10.1200/JCO.20.1.282

[8] Apryl, S.S., Luke, P.A. and Peter, J.A.S. (2009) Risk of Cerebrovascular Events after Neck and Supraclavicular Radiotherapy: A Systematic Review. Radiotherapy and Oncology, 90, 163-165. http://dx.doi.org/10.1016/j.radonc.2008.12.019

[9] Kirova, Y.M., Castro Pena, P., Dendale, R., et al. (2010) Simplified Rules for Everyday Delineation of Lymph Node Areas for Breast Cancer Radiotherapy. The British Journal of Radiology, 83, 683-286. http://dx.doi.org/10.1259/bjr/28834220

[10] Woodward, W.A., Giordano, S.H., Duan, Z., et al. (2006) Supraclavicular Radiation for Breast Cancer Does Not Increase the 10-Year Risk of Stroke. Cancer, 106, 2556-62. http://dx.doi.org/10.1002/cncr.21943

[11] Chung, T.S., Yousem, D.M., Lexa, F.J., et al. (1994) MRI of Carotid Angiopathy after Therapeutic Radiation. Journal of Computer Assisted Tomography, 18, 533-538. http://dx.doi.org/10.1097/00004728-199407000-00003

[12] Fonkalsrud, E.W., Sanchez, M., Zerubavel, R., et al. (1977) Serial Changes in Arterial Structure Following Radiation Therapy. Surgery, Gynecology \& Obstetrics, 145, 395-400.

[13] Elerding, S.C., Fernandez, R.N., Grotta, J.C., et al. (1981) Carotid Artery Disease Following External Cervical Irradiation. Annals of Surgery, 194, 609-615. http://dx.doi.org/10.1097/00000658-198111000-00009

[14] Shariat, M., Alias, N.A.A. and Biswal, B.M. (2008) Radiation Effects on the Intima-Media Thickness of the Common Carotid Artery in Post-Radiotherapy Patients with Head and Neck Malignancy. Postgraduate Medical Journal, 84, 609-612. http://dx.doi.org/10.1136/pgmj.2008.068569

[15] Matthew H.C., Christopher M.G., Carl P.J., et al. (2003) Valvular Dysfunction and Carotid, Subclavian, and Coronary Artery Disease in Survivor of Hodgkin Lymphoma Treated with Radiation Therapy. JAMA, 290, 2831-2837. http://dx.doi.org/10.1001/jama.290.21.2831

[16] Graus, F., Rogers, L.R. and Posner, J.B. (1985) Cerebrovascular Complications in Patients with Cancer. Medicine, 64, 16-35. http://dx.doi.org/10.1097/00005792-198501000-00002

[17] Bilora, F., Pietrogrande, F., Petrobelli, F., et al. (2006) Is Radiation a Risk Factor for Atherosclerosis? An Echo-Color Doppler Study on Hodgkin and Non Hodgkin Patients. Tumori, 92, 295-298.

[18] Bilora, F., Pietrogrande, F., Campagnolo, E., et al. (2009) Are Hodgkin and Non-Hodgkin Patients at a Greater Risk of Atherosclerosis? A follow-up of 3 years. European Journal of Cancer Care, 88, 134-139.

[19] Gianicolo, M.E., Gianicolo, E.L., Tramacere, F., et al. (2010) Effects of External Irradiation of the Neck Region on In- 
tima Media Thickness of the Common Carotid Artery. Cardiovascular Ultrasound, 8, 1-7. http://dx.doi.org/10.1186/1476-7120-8-8

[20] Jephcott, C.R., Tyldesley, S. and Swift, C.L. (2004) Regional Radiotherapy to Axilla and Supraclavicular Fossa for Adjuvant Breast Treatment: A Comparison of Four Techniques. International Journal of Radiation Oncology • Biology - Physics, 60, 103-110. http://dx.doi.org/10.1016/j.ijrobp.2004.02.057

[21] Wang, X., Yu, T.K., Salehpour, M., et al. (2009) Breast Cancer Regional Radiation Fields for Supraclavicular and Axillary Lymph Node Treatment: Is a Posterior Axillary Boost Field Technique Optimal? International Journal of Radiation Oncology • Biology • Physics, 74, 86-91. http://dx.doi.org/10.1016/j.ijrobp.2008.07.016

[22] Lomax, A.J., Cella, L., Weber, D., et al. (2003) Potential Role of Intensity-Modulated Photons and Protons in the Treatment of the Breast and Regional Nodes. International Journal of Radiation Oncology • Biology • Physics, 55, 785-792. http://dx.doi.org/10.1016/S0360-3016(02)04210-4

[23] Vitolo, V., Millender, L., Quivey, J., et al. (2009) Assessment of Carotid Artery Dose in the Treatment of Nasopharyngeal Cancer with IMRT versus Conventional Radiotherapy. Radiotherapy and Oncology, 90, 213-220. http://dx.doi.org/10.1016/j.radonc.2008.08.014 